\title{
„Das stand doch auf Facebook“: Museen in sozialen Netzwerken
}

\author{
Verena Holtkötter ${ }^{1}$, Michael Prilla ${ }^{2}$
}

LWL-Landesjugendamt, Schulen, Koordinationsstelle Sucht, Landschaftsverband WestfalenLippe (LWL) ${ }^{1}$

Informations- und Technikmanagement, Ruhr-Universität Bochum²

\section{Zusammenfassung}

Museen sind zunehmend in sozialen Medien präsent, um sich neue Zielgruppen zu erschließen und Nutzer aktiver in die Museumsarbeit zu integrieren. Gleichwohl haben die Gestalter solcher Auftritte oft kaum Kenntnisse über die Ansprüche ihrer Nutzer. In diesem Beitrag wird eine Studie mit 16 (potenziellen) Nutzern der Auftritte zweier Museen in Facebook beschrieben, anhand derer Nutzerbedarfe identifiziert werden. Der Beitrag benennt hierzu vier Dimensionen (Themen) dieser Ansprüche, leitet eine Nutzertypologie ab und beschreibt Gestaltungsempfehlungen auf Basis der Erkenntnisse aus der Studie.

\section{Einleitung}

Kultureinrichtungen wie Museen sind im Wandel: Einerseits besteht ein fortschreitender Zwang zur inhaltlichen und betriebswirtschaftlichen Rechtfertigung des Daseins von Kulturbetrieben (Hausmann \& Frenzel 2014), andererseits verändern sich sowohl die Interpretation des Bildungsauftrags als auch die Erwartungen von Besuchern der Kultureinrichtungen hin zur Inklusion verschiedener Zielgruppen bzw. zu einer Erlebniskultur (Chung et al. 2014; Lehman \& Roach 2011; Hausmann \& Frenzel 2014). Für beide Stränge dieses Wandels müssen Museen neue Wege finden, mit denen sie auf Besucher eingehen, mit ihnen interagieren und sie an sich binden können. Vermehrt treten Kultureinrichtungen mit ihrem Programm daher auch in sozialen Medien auf.

Die Nutzung sozialer Medien eröffnet für Kultureinrichtungen Potenziale der Kommunikation mit und Bindung von Interessenten, erzeugt gleichzeitig aber auch neue Herausforderungen. Negative Meinungen und Meldungen, positive Nachrichten und Ereignisse können in kürzester Zeit an viele Menschen kommuniziert werden. Kenntnisse über die Ansprüche von Nutzern sind daher auch für ein erfolgreiches Engagement von Kultureinrichtungen in sozialen Medien unerlässlich. Genau diese Notwendigkeit bereitet jedoch vielen Einrichtungen in 
der Praxis Schwierigkeiten: Oft wissen diese nicht, welche Bedarfe und Gewohnheiten ihre Nutzer haben (Chung et al. 2014).

Die Anzahl der Studien zu Ansprüchen an Unternehmen in sozialen Medien wächst beständig; im Kontext von Kultureinrichtungen sind diese jedoch weitgehend unerforscht. Es ist zudem fraglich, ob Nutzeransprüche an Kultureinrichtungen mit denen an Unternehmen einhergehen. Dieser Beitrag beschreibt daher eine Studie, in der die Auftritte zweier Museen in Facebook untersucht werden. Der Beitrag trägt zum Verständnis der Nutzung sozialer Medien für Kultureinrichtungen bei, indem er Nutzerbedarfe und -gewohnheiten identifiziert und daraus resultierende Gestaltungsmöglichkeiten für den Auftritt von Museen und anderen Kultureinrichtungen in sozialen Medien aufzeigt.

\section{Verwandte Arbeiten}

\subsection{Gestaltung und Marketing moderner Kultureinrichtungen}

Im Zuge des gesamtgesellschaftlichen Wertewandels und des steigenden Ökonomisierungsdrucks befinden sich Museen mehr denn je in einem Spannungsfeld zwischen ihren traditionellen, kulturpolitisch vorgegebenen Zielsetzungen und den zunehmend erlebnisorientierten Ansprüchen potenzieller Besucher (Gentischer 2011). Als offene Bildungseinrichtungen "Orte des Lernens" - können Museen die Bedürfnisse der Besucher nicht mehr befriedigen (Heinze 2008). Erlebnisversprechende Angebote (z. B. "Lange Nacht der Museen") sind heute zentraler Bestandteil der kulturellen Vermittlungsarbeit. In diesem Zusammenhang wird auch die Nutzung sozialer Medien für Kultureinrichtungen interessant (Chung et al. 2014; Hausmann \& Frenzel 2014).

\subsection{Organisationen in Facebook: Wirkung und Nutzerbedarfe}

Die Präsenz von Unternehmen in sozialen Netzwerken ist häufig durch ökonomische Nutzerabsichten motiviert (Chung et al. 2014; Heymann-Reder 2012; Weinberg 2012). Dabei stehen bspw. materielle oder konsumrelevante Aspekte wie der Erhalt von Rabatten, Vergünstigungen oder (Sonder-) Angeboten bei den Nutzeransprüchen im Vordergrund (Chung et al. 2014). Weitere Inhalte sind unternehmensbezogene Informationen, der Aufbau eines Markenbewusstseins, die Steigerung der Markensympathie und -verbundenheit, Kundenbindung oder Rekrutierung (Heymann-Reder 2012; Weinberg 2012).

\subsection{Museen in sozialen Netzwerken}

In der Besucherforschung und für das Marketing bieten soziale Netzwerke wie Facebook bedeutsame Potenziale für Kultureinrichtungen. Viele Museen sind bereits in Facebook aktiv bzw. entwickeln Strategien für ihre künftige Beteiligung im sozialen Netzwerk (Chung et al. 2014; Lehman \& Roach 2011; Scheurer \& Spiller 2010; Hausmann \& Frenzel 2014). Im Unterschied zu Organisationen aus der Wirtschaft liegt das Potenzial hierbei nicht vorrangig 
in materiellen Vorteilen (bspw. Vergünstigungen bei Produkten), sondern in nichtmateriellen Bereichen wie der Verbesserung der Beziehung zwischen Besuchern und dem Museum (Chung et al. 2014) oder der besseren Vermittlung von Kunst und Kultur (Hausmann \& Frenzel 2014).

Vorhandene Arbeiten beziehen sich häufig auf den Bildungsauftrag von Museen, also die Vermittlung des Verständnisses für Exponate bspw. durch Bereitstellung von Informationen (Chung et al. 2014). Schwieriger ist häufig die Bindung von Interessenten an und die Gewinnung von Besuchern für eine Einrichtung, da Marketing und Kulturvermittlung in vielen Einrichtungen getrennt sind (Hausmann \& Frenzel 2014). Soziale Netzwerke können eine Möglichkeit des Marketings sein (Lehman \& Roach 2011), die Aufmerksamkeit für die Einrichtung erhöhen (bspw. durch Informationen zu Ausstellungen) und Community-Bildung ermöglichen (Chung et al. 2014). Diese Potenziale entstammen jedoch meist der Befragung von Personen, die für den Auftritt von Kultureinrichtungen verantwortlich sind, und beziehen Nutzer nicht ein. Chung et al. (2014) berichten sogar von Unsicherheiten einiger Befragten über die Wirksamkeit ihres Vorgehens bei der Nutzung sozialer Medien.

Die beschriebenen Arbeiten zeigen zwar die Ziele und Schwierigkeiten von Kultureinrichtungen in sozialen Netzwerken auf, untersuchen jedoch nicht die Bedarfe und Ziele von Nutzern, die Kontakt zu einer Kultureinrichtung haben. Dies führt dazu, dass statt gezielter, nutzerorientierter Strategien häufig Annahmen über die Zielgruppen die Social MediaAktivitäten leiten (Chung et al. 2014; Lehman \& Roach 2011).

\section{Studie: Nutzerbedarfe am Beispiel zweier Museen}

Zur Identifikation von Nutzeransprüchen an den Auftritt von Kultureinrichtungen in sozialen Medien wird im Folgenden eine Studie beschrieben, in der diese Bedarfe und Wünsche untersucht werden. Sie betrachtet Facebook als Beispiel sozialer Netzwerke. In dem weltweit größten sozialen Netzwerk findet sich eine Vielzahl und Bandbreite von Nutzern. Facebook ist die priorisierte Social Media-Anwendung für die überwiegende Zahl von Kultureinrichtungen, die in sozialen Medien präsent sind.

\subsection{Forschungsfrage}

Forschungsziel ist die Identifikation von Nutzererwartungen an Museen in sozialen Netzwerken, um daraus Gestaltungsempfehlungen für den Auftritt von Kultureinrichtungen in sozialen Medien zu gewinnen. Da bisher kaum bzw. keine Erkenntnisse zu diesen Erwartungen vorhanden sind, wird folgende zentrale Forschungsfrage leitend sein: Welche Ansprüche haben Nutzer an die Facebook-Seiten von Museen? Als Teil dieser Frage werden in der Analyse der Kontext der Nutzer, ihr Lese- und Interaktionsverhalten, Nutzungsintentionen sowie die Rahmenbedingungen der Facebook-Seiten thematisiert. 


\subsection{Die Museen}

Gegenstand der Untersuchung sind die Facebook-Seiten des LWL-Museums für Kunst und Kultur in Münster ${ }^{1}$ und des LWL-Museums für Archäologie in Herne ${ }^{2}$. Beide Museen sind seit 2010 und mit gewissem Erfolg in Facebook vertreten, wie ihre Nutzerzahlen (jeweils über 1.500 „Gefällt mir“-Angaben) zeigen, wünschen sich jedoch mehr Resonanz für ihre Seiten und besseren Kontakt mit (möglichen oder vorhandenen) Besuchern. Es wurden bewusst Museen mit unterschiedlichen Themenbereichen und aus verschiedenen Regionen gewählt, um einen Einfluss beider Faktoren in der Untersuchung gering zu halten.

\subsection{Untersuchungsdesign und Ablauf der Studie}

Die Ergebnisse vorhandener Studien zum Auftritt von Unternehmen in sozialen Medien können für Kulturbetriebe nicht ohne Weiteres übernommen werden. Zum einen weichen die Spezifika von Kulturbetrieben wie bspw. öffentlich-rechtliche Trägerschaft, qualitative Zielsetzungen und kultureller Bildungsauftrag von den Zielen eines Unternehmens ab, zum anderen verfolgen Kulturbetriebe in sozialen Medien eine nicht-materiell orientierte Strategie (Chung et al. 2014). Statt eines hypothesenprüfenden, quantitativen Verfahrens bedient sich die Studie daher eines qualitativen Ansatzes, in dem eine Stichprobe aus (potenziellen) Nutzern der Museen interviewt wurde (siehe Auswahl der Teilnehmer unten). In semistrukturierten Interviews wurden Fragestellungen zu Lese- und Interaktionsverhalten auf den Seiten, Nutzungsintentionen, inhaltlichen und formalen Gestaltungsaspekten thematisiert. Um die Ableitung eines Modells von Nutzerbedarfen aus dem Datenmaterial zu ermöglichen, erfolgte die Auswertung der Studie entlang der von Glaser \& Strauss (1968) entwickelten Grounded Theory ${ }^{3}$. In der offenen Kodierung wurden Codes unmittelbar aus den transkribierten Interviews entwickelt und zu Kategorien zusammengeführt. Das daraus resultierende Kategorienschema wurde sukzessive anhand weiterer Fälle so lange modifiziert und weitergeführt, bis ein ausreichender Sättigungsgrad erreicht war (Kelle \& Kluge 2010).

\subsection{Die Teilnehmer}

Die Rekrutierung der Untersuchungspersonen erfolgte zunächst auf den zu untersuchenden Facebook-Seiten selbst. Unter Berücksichtigung der Facebook-Nutzungsbedingungen wurde mithilfe eines Gewinnspiels (Verlosung von Eintrittskarten) unter den Seiten-Nutzern zur Teilnahme aufgerufen und ein Link zu einer externen Anmeldeplattform bereitgestellt.

1 https://www.facebook.com/LWLMuseumKunstundKultur

2 https://www.facebook.com/LWLMuseumHerne

3 In vielen Kodierungsansätzen wird die Kodierung durch zwei oder mehr unabhängige Personen durchgeführt, um eine Objektivierung der Ergebnisse zu erreichen. Die Grounded Theory als Interpretationsmethode weicht von solchen Ansätzen ab und verwendet bspw. vorläufige Codes und Memos, die einer Übereinstimmungsprüfung zwischen Kodierern nicht wie bei anderen Methoden zugeführt werden können. Entsprechend wurde die Kodierung in der vorliegenden Studie durch eine Forscherin durchgeführt. 
Für die Auswahl der Stichprobe aus den wie oben rekrutierten Freiwilligen wurden aus verschiedenen möglichen Merkmalen aus der Literatur die Merkmale „Aktivitätsgrad“ und „fachspezifischer Interessengrad“ ausgewählt. Das Aktivitätskriterium erfasst die in sozialen Medien vorhandene Heterogenität passiv konsumierender und aktiv partizipierender Nutzer (Haas 2007), das individuelle Interesse am jeweiligen Fachgebiet eines Museums (hier: Archäologie bzw. Kunst) ist für die Rezeption von Angeboten durch (potenzielle) Besucher entscheidend (Heinze 2008). Durch die Kombination dieser Merkmale wurde in der Stichprobe eine Verknüpfung der Nutzer von Facebook-Seiten und Museumsbesuchern hergestellt. Die Merkmale erhielten hierfür eine vereinfachte Einteilung in zwei Ausprägungen (hoch / niedrig) mit festgelegten Definitionsbereichen. Die Stichprobe wurde zunächst auf Nutzer beschränkt, die bereits durch eine „Gefällt mir"-Angabe mit den Seiten verbunden sind. Die Kriterien der Fallauswahl ließen sich nur im Dialog mit den einzelnen Untersuchungspersonen identifizieren, um über den tatsächlichen Bedarf des Interviews zu entscheiden. Die auf Basis des Vorgesprächs getroffene Fallzuordnung wurde im Kontext des gesamten Interviewmaterials im Nachgang nochmals überprüft. Während der Stichprobenziehung wurde kontinuierlich auf eine ausgewogene Zusammensetzung hinsichtlich soziodemografischer Merkmale geachtet (Alter, Geschlecht, Bildung, Beruf, geografische Nähe zum Museum). So wurden entlang des beschriebenen Stichprobenplans 14 Teilnehmer für die Studie gewonnen. Ergänzend wurden zwei Teilnehmer in die Untersuchung aufgenommen, die Erfahrungen mit Facebook-Seiten von Museen haben, jedoch keine der beiden Seiten aus der Studie abonniert hatten. Durch ihren Einbezug ist das Untersuchungsfeld weiter erschlossen worden. Insgesamt setzt sich das Erhebungsmaterial aus den Aussagen von 16 Untersuchungspersonen zusammen.

\section{Ergebnisse}

Aus der Auswertung wurden vier Themen mit zehn Hauptkategorien und Unterkategorien gewonnen. Diese beschreiben die erforschten Nutzeransprüche an die Facebook-Seiten der Museen. Auf Basis des Stufenmodells der empirisch begründeten Typenbildung nach Kluge (1999) bildeten diese Kategorien die Grundlage der unten beschriebenen Nutzertypologie.

\subsection{Nutzeransprüche: Kategorien}

Die Ansprüche von Nutzern lassen sich anhand der Themen Nutzerkontext, Nutzungsverhalten, Nutzungszweck und Nutzungsrahmen differenzieren. Im Folgenden werden diese beschrieben und mit Zitaten aus den Interviews illustriert.

Der Nutzerkontext lässt sich in das Interesse am Fachgebiet und Bezugspunkte zum Museum differenzieren. Das Interesse kann gering (,„dass man halt ab und an mal ins Museum geht“) oder stark (,Ich habe selber Archäologie studiert“) sein, Bezugspunkte sind lokale Nähe (,,das war immer schon in der Nähe irgendwie“); persönlicher Kontakt („Das ist natürlich noch mal was anderes, wenn man eine persönliche Verbindung hat zu den Leuten.") und eigene Besuche (,,ich kenne das Museum schon sehr lange“). 
Das Nutzungsverhalten lässt sich in Rezeptionsgewohnheiten, Rezeptionsbedarf, Inhaltliche Präferenzen, Interaktionsintensität, Interaktionsgewohnheiten und Interaktionsantreiber und -barrieren aufschlüsseln. Neben flüchtiger Wahrnehmung konnten Rezeptionsgewohnheiten wie umfassende (,Also, ich gucke eigentlich täglich einmal rein und sobald ich dann zuhause bin und Zeit habe, dann beschäftige ich mich auch damit") oder selektive Wahrnehmung (,Also, ich gehe jetzt nicht gezielt auf die Seite. Aber wenn ich Beiträge sehe (...), dann gucke ich mir die eigentlich auch an. ") identifiziert werden; bei den Rezeptionsbedarfen hielten sich regelmäßige und unregelmäßige Nutzung die Waage. Die inhaltlichen Präferenzen der Nutzer gehen jedoch stark auseinander: Einige Nutzer sind an Neuigkeiten (,,ich würde gerne mehr erfahren über das aktuelle Programm") oder Unterhaltung interessiert, während andere Impressionen aus dem Museum (,,ich kriege Einblicke auch hinter Kulissen“) oder weiterführende Inhalte (,dass das Museum Informationen über sich präsentiert, aber auch über andere Themen aus der Archäologie") bevorzugen. Gleichzeitig lehnen einige Nutzer Inhalte ab, wenn diese über die Exponate des Museums hinausgehen (,,das hat jetzt nichts direkt mit dem Museum zu tun. ").

Hinsichtlich der Interaktionsintensität nutzt die Hälfte der Befragten die Seiten aktiv und intensiv (lesen und Beiträge schreiben), die andere Hälfte sporadisch oder passiv. Die Interaktionsgewohnheiten schließen Funktionen wie „Gefällt mir“ als Zustimmung und „Teilen“ ebenso ein wie Kommentare (,Und ich schreibe auch gerne Kommentare oder stelle auch mal eine Frage“) und eigeninitiierte Aktionen (,wenn ich selber etwas entdecke (...), dass man das dann einfach mal mit auf die Timeline ,postet“"). Als Interaktionsantreiber wurden Wertschätzung der Arbeit (,,man muss es ein bisschen auch wertschätzen“), soziale Kontakte $\mathrm{zu}$ anderen Nutzern (,Man hat halt Leute mit Fachwissen und Interesse und kann die sofort erreichen") und Aufmerksamkeit (,dass man gesehen wird“) sowie persönlich relevante Inhalte und persönlicher Zugang (,durch das Internet habe ich halt die Möglichkeit, wirklich Ansprechpartner zu bekommen") genannt. Als Interaktionsbarrieren nannten die Nutzer Zweifel (, ich hätte da das Gefühl, ich könnte gar nicht mitreden“), mangelnde Identifikation mit der Masse anderer Nutzer (,wenn da einer stumpf drunter schreibt, , voll geil", oder so, dann ist das eben plump. “) und Datenschutz- Bedenken (,nicht dass dann hinterher wirklich jeder alles über mich weiß. “).

Für den Nutzungszweck lassen sich als Motive Informationsbeschaffung (,was die gerade so machen und wo die gerade so dran sind“), Freizeitplanung (,,damit man es nicht verpasst und gleich daran erinnert wird"), Teilhabe an der Museumsarbeit (,da kommt was Menschliches rüber"), Gemeinschaftserlebnis (,,mit Gleichgesinnten in Kontakt zu kommen“), Unterhaltung (,Da war so ein Rätsel verknüpft mit der Homepage“) und Exklusivität der Informationen (,,dass man da Infos bekommen hat, die man halt nicht aus der Zeitung oder dem Radio bekommen hat. Wo ich dann gesagt habe, ,ach Mensch, das stand doch auf Facebook' (...) ") finden.

Der Nutzungsrahmen beschreibt Voraussetzungen für die Nutzung wie aufbereitete Inhalte (Aktualität, Multimedia, etc.), eine benutzerfreundliche Bedienung (auch auf Mobilgeräten) und eine ansprechende Optik. In dieser Kategorie ist die Varianz gegenüber den anderen Kategorien deutlich geringer, sodass hier vermutet werden kann, dass es sich um allgemein (und auch für andere Anwendungen) gültige Hygienefaktoren handelt. 
Die Vielfalt der Ansprüche sowie ihrer Ausprägungen weist darauf hin, dass es nicht einen Typ von Nutzern der Facebook-Seiten von Museen gibt. Entsprechend bestimmen individuelle Faktoren, welche Inhalte der Facebook-Seite eines Museums für Nutzer interessant sind; der individuelle Mehrwert steht im Vordergrund. Es ist daher eine Differenzierung von Nutzertypen notwendig, um Nutzeransprüchen (gezielt) zu begegnen.

\subsection{Typologie von Nutzern: Ein Vorschlag}

Die Gruppierung der Befragten anhand ihrer gemeinsamen Eigenschaften ermöglicht die Entwicklung einer Nutzertypologie. Unter Rückbezug auf die Kriterien der Fallauswahl als zentrale und indizierte Differenzierungsmerkmale (fachspezifischer Interessengrad, Aktivitätsgrad) können die teils konträren Themen und Kategorien in vier Nutzertypen zusammengeführt werden. Bei den beiden befragten potenziellen Seiten-Nutzern ließen sich keine nennenswerten Abweichungen in den Ansprüchen verzeichnen, sodass ihre Aussagen in die Nutzertypologie eingebunden werden konnten. In der Typologie wird jedem Nutzertyp in Tabelle 1 ein Name zugeordnet, der plakativ für den Nutzungszweck steht. Die Intensität von Interesse und Nutzung wächst von links unten nach rechts oben.

\begin{tabular}{|c|c|c|}
\hline Niedrig & $\begin{array}{l}\text { Nachrichtenkonsument (5): } \\
\text { Fachliche, weiterführende Informati- } \\
\text { onen, passive Nutzung, Versorgung } \\
\text { mit Inhalten }\end{array}$ & $\begin{array}{l}\text { Mitgestalter (5): } \\
\text { Intensive Nutzung, Kommentare und } \\
\text { eigene Beiträge, Informationen und } \\
\text { Interaktion zur Vernetzung }\end{array}$ \\
\hline \multirow[t]{2}{*}{ Niedrig } & $\begin{array}{l}\text { Freizeitplaner (3): Besucherinforma- } \\
\text { tionen, kaum Interaktion, beiläufige } \\
\text { Nutzung von Inhalten, sporadische } \\
\text { Informationen }\end{array}$ & $\begin{array}{l}\text { Museumssympathisant (3): } \\
\text { Bedarfsweise Rezeption, Interaktion } \\
\text { als Wertschätzung, Informationen } \\
\text { zur Teilhabe am Geschehen }\end{array}$ \\
\hline & Niedrig & Aktivität \\
\hline
\end{tabular}

Tabelle 1: Nutzertypologie - Die Nutzer der untersuchten Facebook-Seiten von Museen. In Klammern: Anzahl der Personen, die der Rolle in der Stichprobe zugeordnet wurden.

Der Typ Freizeitplaner beschreibt Nutzer, für die sporadisch Besucherinformationen interessant sind. Die Seiten sollen zu einem Museumsbesuch anregen. Freizeitplaner sind neugierig auf aktuelle Veranstaltungen, beteiligen sich aber (auch wegen Zweifeln an ihren Beiträgen) kaum aktiv auf den Facebook-Seiten der Museen.

Der Typ Nachrichtenkonsument beschreibt Nutzer, die ein hohes fachliches Interesse am Museum haben, die Seite jedoch hauptsächlich rezipierend nutzen. Für sie ist die Seite ein Medium, um aktuelle Informationen des Museums und des Fachgebiets zu erhalten. Eine aktive Beteiligung scheitert an der mangelnden Identifikation mit der Masse anderer Nutzer.

Der Typ Museumssympathisant beschreibt Nutzer, die ohne spezifisches Interesse auf den Facebook-Seiten agieren, den direkten Kontakt zum Museum suchen, aber wenig Interesse 
an anderen Nutzern haben. Sie sehen in der Seite eine Möglichkeit zur (virtuellen) Teilhabe an der Museumswelt. Der persönliche Zugang zum Museum ist ihnen wichtig, Kommentare werden häufig zur Wertschätzung oder Zustimmung gegenüber dem Museum erstellt.

Der Typ Mitgestalter beschreibt Nutzer, die aus einem fachlichen Interesse heraus regelmäBig Inhalte konsumieren und zudem auch erstellen. Für sie stehen das Gemeinschaftserlebnis und die Vernetzung untereinander im Vordergrund. Durch ihr aktives Mitwirken tragen sie engagiert zur Gestaltung der Seite bei.

Den Autoren ist bewusst, dass diese Typologie angesichts der Vielfalt der oben beschriebenen Merkmale eine Vereinfachung der Betrachtung von Nutzern der untersuchten FacebookSeiten darstellt. Sie kann dennoch dazu dienen, die Komplexität der Bedarfe darstellbar zu machen und für die Unterschiede bei diesen Bedarfen zu sensibilisieren. Sie kann daher sowohl in der praktischen Gestaltung der Auftritte von Museen und anderen Kulturbetrieben als auch für weitere Forschungen als Grundlage dienen.

\section{Diskussion}

\subsection{Die Ergebnisse im Kontext vorhandener Untersuchungen}

Die Studie kann Erkenntnisse aus verwandten Studien zu Unternehmen in Teilen bestätigen, erweitert sie jedoch um bedeutsame Komponenten. Übereinstimmende Ergebnisse finden sich in dem Anspruch, in sozialen Netzwerken Informationen über die jeweilige Organisation erhalten zu wollen, sowie in den Verbundenheits- und Sympathie-Aspekten.

Neue Erkenntnisse für den Gegenstandsbereich von Museen finden sich in der Breite des Informationsanspruchs von Nutzern. So weckt ihre Position im Freizeitsektor Bedarfe nach Informationen zur Freizeitplanung (bspw. Veranstaltungstermine); Nutzer mit (gemeinsamem) fachspezifischem Interesse erwarten Informationen über das jeweilige Fachgebiet des Museums. Facebook-Seiten von Museen werden auf diese Weise zu einer Austausch- und Informationsplattform und zu einem Treffpunkt für Gleichgesinnte.

In verwandten Studien zu Unternehmen ist die interaktive Beteiligung auf materielle Aspekte und den Kontakt zum Unternehmen konzentriert. Derartige ökonomische Nutzeransprüche finden sich in der vorliegenden Studie nicht. Insgesamt zeigen die Nutzeransprüche an $\mathrm{Mu}$ seen innerhalb sozialer Netzwerke einen eher inhaltlichen Charakter. Immaterielle Interessen wie Freizeitplanung, Austausch oder Gemeinschaft stehen im Vordergrund.

\subsection{Gestaltung von Facebook-Seiten für Museen: Vorschläge}

Aus der Studie lassen sich zur inhaltlichen Gestaltung und der Interaktionsgestaltung Empfehlungen ableiten. Hinsichtlich der inhaltlichen Gestaltung wird deutlich, dass die Heterogenität der Nutzergruppen verschiedene Inhalte notwendig macht, wenn ein Museum alle Gruppen ansprechen möchte. So sind sowohl allgemeine Inhalte wie Informationen über den laufenden Betrieb, Besucherinformationen wie Hinweise aus dem aktuellen Ausstellungs- 
programm als auch Museumsimpressionen wie Einblicke in den Museumsbetrieb nachgefragt. Ein Teil der Nutzer interessiert sich zudem für spezielle und weiterführende Informationen (bspw. fachspezifische Informationen), die jedoch von anderen Nutzern eher abgelehnt werden. Dies kann bei der inhaltlichen Gestaltung berücksichtigt werden, in dem bspw. weiterführende Inhalte lediglich verlinkt werden, oder indem eine Gruppe für Interessierte an weiterführenden Inhalten erstellt wird.

Für die Interaktionsgestaltung sind neben relevanten und diskussionsfähigen Inhalten auch die persönlich wahrgenommene Atmosphäre und Unterstützung bei der aktiven Nutzung von Facebook-Seiten entscheidend. Nutzer, die sich bisher nicht äußern, weil sie an der Qualität ihrer Äußerungen zweifeln oder sich mit der Masse anderer Benutzer nicht identifizieren können, sollten aktiv unterstützt werden, wenn das Museum ihre Mitgestaltung wünscht hier liegen Potenziale, insbesondere in der Aktivierung von Nachrichtenkonsumenten, die zwar Interesse zeigen, aber (noch) nicht aktiv mitgestalten. Gelegenheiten zur Äußerung wie bspw. offenes Feedback nach einem Besuch können hier eine kommunikationsoffene Atmosphäre schaffen. Die in der Studie gewonnenen Erkenntnisse können bei der Auswahl solcher Möglichkeiten unterstützen. Auffällig ist weiterhin, dass Mechanismen zur (anschlussfähigen) Kommunikation hauptsächlich für den Typ des Mitgestalters relevant sind; Sympathisanten nutzen diese lediglich für einfaches Feedback. Gleichwohl erzeugen diese Mechanismen den Eindruck einer aktuellen, häufig genutzten Seite, und dies wird von Nachrichtenkonsumenten und Freizeitplanern wahrgenommen. Bei der Gestaltung und Pflege von Seiten für Kultureinrichtungen kann sich daher die Förderung der Beteiligung von Mitgestaltern auch auf die Zahl der Vertreter anderer Typen auswirken.

\subsection{Integration von Nutzern in Museumsarbeit}

Die Studie zeigt Potenziale auf, mit denen Nutzer enger in die Museumarbeit integriert werden können. So sind für Nachrichtenkonsumenten, Mitgestalter und Museumssympathisanten Museumsimpressionen und der persönliche Zugang zu Inhalten und Ansprechpartnern eine wichtige Motivation zur Nutzung der Facebook-Seiten. Eine solche Transparenz und Sichtbarkeit der Arbeits- und Gestaltungsprozesse eines Museums kann diese Gruppen an die Facebook-Seiten binden. Zudem kann die Verbindung der Nutzertypen mit Möglichkeiten der aktiven Beteiligung Nutzer in die Gestaltung des Museums einbinden: Während Mitgestalter bereits Inhalte einbringen, könnten Sympathisanten durch Abstimmungen bzw. Feedback und Nachrichtenkonsumenten durch die oben beschriebenen Gruppen an die aktive Mitgestaltung herangeführt werden.

\section{Zusammenfassung und Ausblick}

Der Beitrag zeigt in einer Studie mit 16 Facebook-Nutzern auf, dass Kultureinrichtungen vielfältige Nutzeransprüche bewältigen müssen, die über die aus Studien mit Seiten von Unternehmen in sozialen Netzwerken bekannten Ansprüche hinausgehen. Er zeigt zudem, dass die Kenntnis dieser Ansprüche großes Potenzial für die Bindung von Interessenten und 
deren Mitgestaltung birgt. Mit Blick auf die zentrale Forschungsfrage nach Nutzeransprüchen an Museumspräsenzen in sozialen Netzwerken zeigt sich, dass verschiedene Nutzertypen und ihr persönlicher Nutzungszweck betrachtet werden müssen. Als zentrale Beiträge wurden hierzu Kategorien von Nutzeransprüchen, eine Typologie von Nutzern sowie daraus resultierende Gestaltungsmöglichkeiten beschrieben.

Grenzen der Untersuchung bestehen in der Vernachlässigung anderer Medien (Twitter, Youtube), anderer Anspruchsgruppen (Sponsoren, Künstler, Presse, Kommunen) und weiterer Museumstypen (Naturkunde, Technik). Zudem betrachtet die Studie vorhandene Nutzer der Facebook-Seiten der Museen bzw. Nutzer mit Erfahrungen auf ähnlichen Seiten und kann daher lediglich erste Aufschlüsse zur Gewinnung neuer Nutzer geben. Sie weist jedoch auf Inhalte hin, die für verschiedene Nutzer interessant sind, und kann durch anknüpfende Forschungen um die aufgezeigten Bereiche erweitert werden. Die vorliegenden Ergebnisse und die Vorgehensweise der Studie können dabei unterstützend wirken.

\section{Literaturverzeichnis}

Chung, T.-L., Marcketti, S. \& Fiore, A.M. (2014). Use of social networking services for marketing art museums. Museum Management and Curatorship. 29(2), 188-205.

Gentischer, M. (2011). Museumsmarketing: Fallstudie am Beispiel des Stuttgarter Stadtmuseums. Springer-Verlag.

Glaser, B.G. \& Strauss, A.L. (1968). The discovery of grounded theory. Aldine Chicago.

Haas, S. (2007). Web 2.0: Nutzung und Nutzertypen. Eine Analyse auf der Basis quantitativer und qualitativer Untersuchung. Media-Perspektiven.(4), 215-222.

Hausmann, A. \& Frenzel, L. (eds.) (2014). Kunstvermittlung 2.0: Neue Medien und ihre Potenziale. Springer Fachmedien Wiesbaden.

Heinze, T. (2008). Kultursponsoring. Kultursponsoring, Museumsmarketing, Kulturtourismus., 75-87. VS Verlag für Sozialwissenschaften.

Heymann-Reder, D. (2012). Social Media Marketing. Addison-Wesley Verlag.

Kelle, U. \& Kluge, S. (2010). Vom Einzelfall zum Typus: Fallvergleich und Fallkontrastierung in der qualitativen Sozialforschung. VS Verlag.

Kluge, S. (1999). Empirisch begründete Typenbildung-Zur Konstruktion von Typen und Typologien in der qualitativen Sozialforschung [Building types empirically based-Towards the construction of types and typologies in the qualitative social sciences]. Opladen: Leske+ Budrich.

Lehman, K. \& Roach, G. (2011). The strategic role of electronic marketing in the Australian museum sector. Museum Management and Curatorship. 26(3), 291-306.

Scheurer, H. \& Spiller, R. (2010). Kultur 2.0. Neue Web-Strategien für das Kulturmanagement im Zeitalter von Social Media.

Weinberg, T. (2012). Social Media Marketing: Strategien Fur Facebook, Twitter \& Co. O'Reilly Germany. 